Liss C. Werner

\title{
HUNCH 1972: A Second Experiment in Sketch Recognition or: 'I Know the Concept of Your Concept of Interpolation'
}

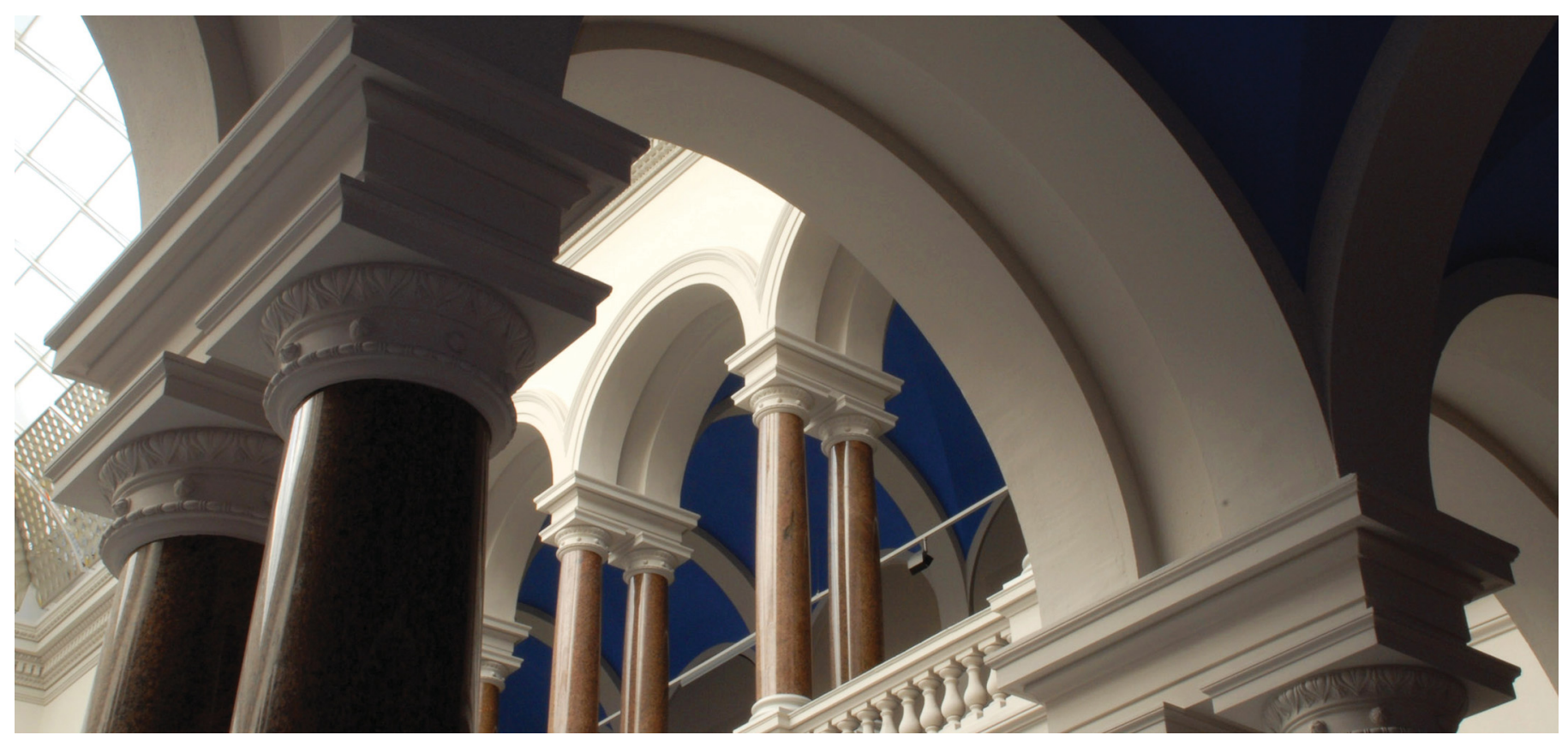

Werner, L. C. (2018). HUNCH 1972: A Second Experiment in Sketch Recognition or: 'I Know the Concept of Your Concept of Interpolation'. In Graphic Imprints (pp. 3-11). Springer International Publishing. https://doi.org/10.1007/978-3-319-93749-6_1 
First published in Werner, L. C. HUNCH 1972: A Second Experiment in Sketch Recognition or: 'I Know the Concept of Your Concept of Interpolation. In: Marcos, Carlos L. (ed.): Graphic Imprints: The influence of Representation and Ideation Tools in Architecture. Proceedings XVII EGA International Publishing, 2018, p. 3-11. 978-3-319-93748-9 ISBN (print).

HUNCH 1972: A Second Experiment in Sketch Recognition or: 'I know the concept of your concept of interpolation'

Liss C. Werner (1)

Technical University Berlin 
First published in Werner, L. C. HUNCH 1972: A Second Experiment in Sketch Recognition or: 'I Know the Concept of Your Concept of Interpolation. In: Marcos, Carlos L. (ed.): Graphic Imprints: The influence of Representation and Ideation Tools in Architecture. Proceedings XVII EGA International Publishing, 2018, p. 3-11. 978-3-319-93748-9 ISBN (print).

\begin{abstract}
.
James Taggart developed the sketch recognition system HUNCH at MIT in 1972. Rather than the user trying to understand the software in order to progress a drawing, in the case of $\mathrm{HUNCH}$, the software observed the user sketching. It enabled a conversation between user and software through the sketch as a medium. HUNCH was one component of the Architecture Machine, created in the Architecture Machine Group (Arch Mac), run by Nicholas Negroponte at MIT between 1967 and 1985 and a brainchild of cross-fertilization between architecture, computer sciences and cybernetics in the early 1970s. One of HUNCHs objectives was to enable even the layman to 'design' a dream home. The paper presents HUNCH's concept as AIequipped 'partner' for the architect, designer or layman.
\end{abstract}

Keywords. HUNCH, sketch recognition, drawing, cybernetics, Architecture Machine Group, graphical interface, Gordon Pask

\section{Introduction}

Since the early 2000s, architecture schools have started extending and partially replacing their classes in architectural design and drafting with architectural research by making, digital crafting and scripting-focusing on trans-disciplinary enterprises and joint-ventures. Architectural education and practice is in a transitional mode of rethinking architectural theory, tools, applications, processes and states of representation and visualization. The latter bears the debate of intuitiveness to the designer's idea and to that of a natural and true translation of the designer's input data into a digital system and subsequently the systems' output data; this can range from an undefined sketch, via a crafted detail to input in form of a scripting language, descriptive modeling, voice recognition or the tracing of physical body movement to be translated into 2d-form or 3-dimensional space. Digital translation tools stem from inventions created and explored in the age of digital archeology (Lynn, 2013); to a large extent in the 1960s and 1970s, extensively developed at MIT under Nicholas Negroponte. Then and now, an integration of trainable artificial intelligence into software systems is necessary if they are targeted at assisting the user, learning about the user, and offering alternative decisions integrating a participative approach. In the paper "An Experiment in Sketch Recognition" Nicholas Negroponte stated that "Since 1967, the authors have argued that computer-aided design is an issue of artificial intelligence, that machines have to understand the meaning of decisions in order to be partners in making them. We have advocated the exploration of artificial intelligence and have condemned computerized solution generation which does not consider it." (Negroponte, 1972) Research projects, that led to the sketch recognition system $\mathrm{HUNCH}$ were components of the Architecture Machine developed in the Architecture Machine Group. They included URBAN5 (Fig. 1), U-Design and SEEK. SEEK was part of a physical environment as a model for an interactive mega-structure, based on the idea of Yona Friedman's mega-grid in the Ville Spatiale during the Metabolism movement. It was a robot arm programmed as positioning device operating in a $1.5 \mathrm{~m} \times 2.4 \mathrm{~m}$ large glass box filled with stacked cubes interacting with a herd of gerbils. The Gerbil project investigated in the relationship between an artificially constructed landscape made of 480 small cubes and a colony of gerbils. The robot arm would reconstruct the block-scape constantly while the gerbils would also change the configuration. SEEK's program was meant to recognize the gerbils' behavioral pattern and rearrange the blocks accordingly. The gerbils participated in the design process.

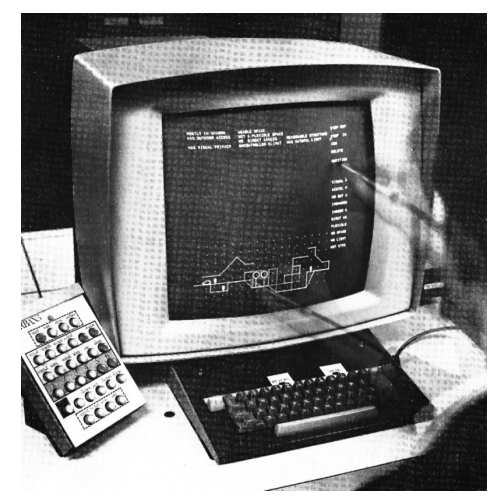

Figure 1. URBAN 5's overlay and the IBM 2250 model 1 cathode-ray-tube used for URBAN 5 (source: openarchitectures.com) 
First published in Werner, L. C. HUNCH 1972: A Second Experiment in Sketch Recognition or: 'I Know the Concept of Your Concept of Interpolation. In: Marcos, Carlos L. (ed.): Graphic Imprints: The influence of Representation and Ideation Tools in Architecture. Proceedings XVII EGA International Publishing, 2018, p. 3-11. 978-3-319-93748-9 ISBN (print).

\section{HUNCH}

In 1972 the sketch recognition system $\mathrm{HUNCH}$ was conceived as "front end for any system" for "Computer Aids to Participatory Architecture" funded by the NSC (National Science Foundation). It was a successor of Sketchpad, by Ivan Sutherland (1963), and URBAN5. The latter became a core element of James Taggart's work in the 1970s was funded through a grant by the Graham Foundation for Advanced Study in the Fine Arts and MIT. "HUNCH - An Experiment in Sketch Recognition" (Negroponte, 1972) was a brain child of Nicholas Negroponte's Architecture Machine Group affected and influences by the British cybernetician Gordon Pask. Theodora Vardouli emphasizes on the cybernetics principles applied at the Architecture Machine Group. She states that "What is unique in the case of Soft Architecture machines is that they are the result of a cross-fertilization of the visions of the golden age of Artificial Intelligence at MIT and the vibrant ideas of Cybernetics, with European influences, namely the work of the "eccentric" Yona Friedman and Gordon Pask." (Vardouli, 2011) HUNCH, like all other components designed by the ArchMac was part of a cybernetic system between architecture, behavioral cognition, construction, and learning HUNCH's expertise was translating individual sketches into drawings through data compression (Fig. 2a and 2b).

\subsection{The Concept of sketching}

$\mathrm{HUNCH}$ was designed as a participant in the architectural from-sketch-to-drawing-process, driven by decision-making capabilities based on heuristics and 'experience', algorithmically supported. The ambiguity between the real thing, the constructed artifact and the concept of it or its construction is the real obstacle to overcome when transferring an architectural vision (purely formal or systemic) from one medium to the other. At this instance it is irrelevant if the medium to transfer the thought, the act of knowing is a piece of paper or a computer disc as a storage device and if one uses a pencil or a set of keys pressed in a certain combination and sequence. The forces at work, during the process of sketching, are made of the instructions the brain gives the hand through the eye. Those instructions are however unimaginably intricate, overly complex and complicated at the same time. The way the human hand and fingers operate a pen has a multitude of parameters at work. The sketch is the medium, the interface between the designer and him or herself, constantly mapping his or her sketch of a final product against the virtual imagined vision. The sketch creates the difference. The recursive act of sketching is an operation of and for design. The form and aesthetics of a final project, a final piece of architecture become clearer and more precise with each iteration of repetition, with each rethinking of the design, reworking, re-sketching, re-entering into the cycle. (Brown, 1969) Each cycle of observation, of observing a conversation between the sketch and the design steers the design process until sketch and vision match. Designer and design vision eventually converge. HUNCH was developed for architects and designers, the sketch recognition problem was ideal for 'testing' the program.

\subsection{The Concept of HUNCH}

HUNCH's goal was to be an acquaintance of a kind and to learn how the parties interacting with it operated. The human hand sketch was a vehicle for training. The question if the act of sketching can actually by dissected into small elements, that through the correct code can again be reconstructed into the behavior of the human hand and thus into the same sketch is rather intriguing. HUNCH was programmed with Gordon Pask's model of human-computer interaction in mind, based on his Conversation Theory (CT) (Pask, 1976), and in line with second order cybernetics (SoC), mainly developed by Heinz von Foerster (Foerster, 1979), Margaret Mead (Mead, 1968) and a number of cyberneticians in the 1960s and 1970s derived from the Macy Conferences held between 1941 and 1960. It followed the model of recursion of information exchange between a sketch and a sketcher. (Werner, forthcoming) The program was built on the combination of three levels that were implemented according Gordon Pask's advice in a private conversation between Nicholas Negroponte and Gordon Pask (Negroponte, 1972):

Level 01: HUNCH's model of the user Level 02: HUNCH's model of your model of it Level 03: HUNCH's model of your model of its model of your model 
First published in Werner, L. C. HUNCH 1972: A Second Experiment in Sketch Recognition or: 'I Know the Concept of Your Concept of Interpolation. In: Marcos, Carlos L. (ed.): Graphic Imprints: The influence of Representation and Ideation Tools in Architecture. Proceedings XVII EGA International Publishing, 2018, p. 3-11. 978-3-319-93748-9 ISBN (print).

HUNCH's level 01-its model of the user-was based on two overriding parameters: speed and pressure. Speed and pressure delivered one part of the information about the user, another part was embedded in the location of each pressure point of the sketch, described as coordinates $\mathrm{X}, \mathrm{Y}$ and $\mathrm{Z}$. Only if the models mentioned on level 03 synchronized, or were close to being synchronized, the two - computer and human - knew each other. HUNCH could understand, which parts of the sketch were clear to its creator and which ones he or she would be in doubt of. HUNCH, subsequently, would help to clarify, merge and marry the architect with his sketch until both matched and finally became a drawing, useful for the production of architecture.

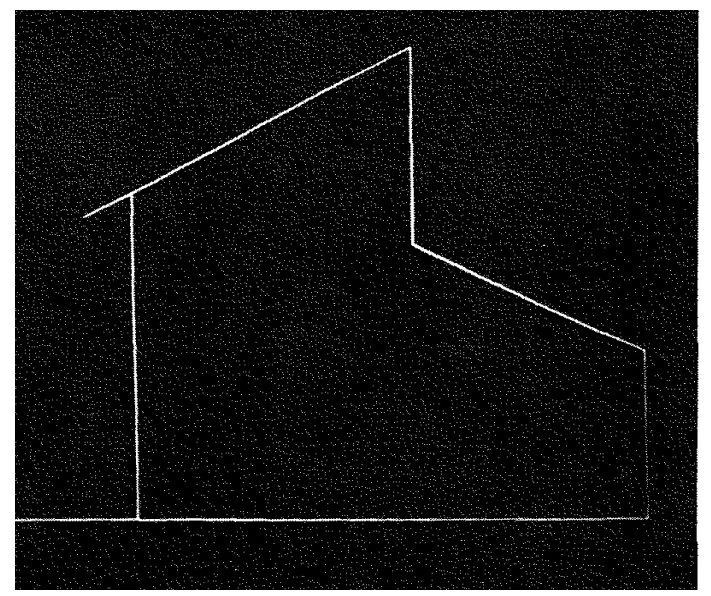

Fig.2a shows a 'raw' sketch before the sketch recognition operation

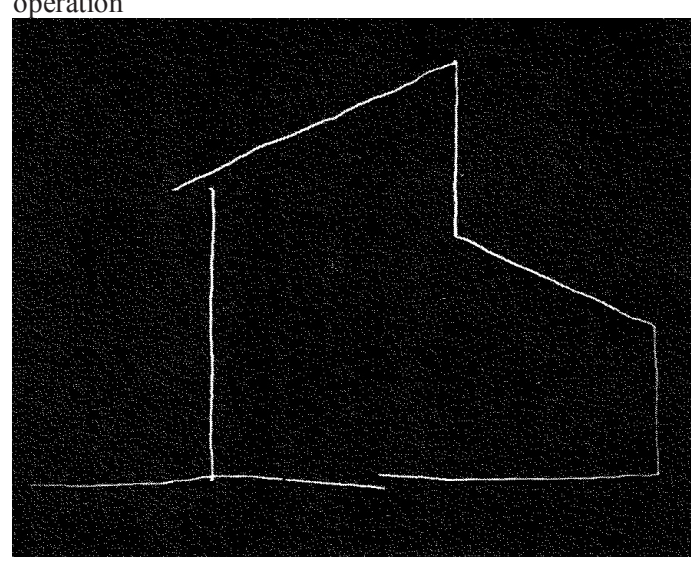

Fig. 2b shows rationalization through data compression of 4128 'core locations' in figure $2 \mathrm{a}$ to 128 'core locations' in figure 2b. See figure 11 and 12 in "HUNCH - An Experiment in Sketch Recognition”, (Negroponte, 1972)

Besides the challenge to recognize sketching patterns, differentiate between an opening and a set of lines which were just sloppily not properly closed, between a curve and an intentional radius, an orthogonal or perspective projection, a crossing or overlapping and to transfer scribbles into clean two and threedimensional geometry, HUNCH could 'sense' pressure and density of lines.

Taggart introduced the B-spline technique to the rationalization operation. Since the regulating point of a spline does lie beyond the curve and not on top, the data input from the user could be subjected to a regulating operation. Figures $3 \mathrm{a}$ and $3 \mathrm{~b}$ show how "varying the order of the spline" (Negroponte, 1973) could change the form of a spline.
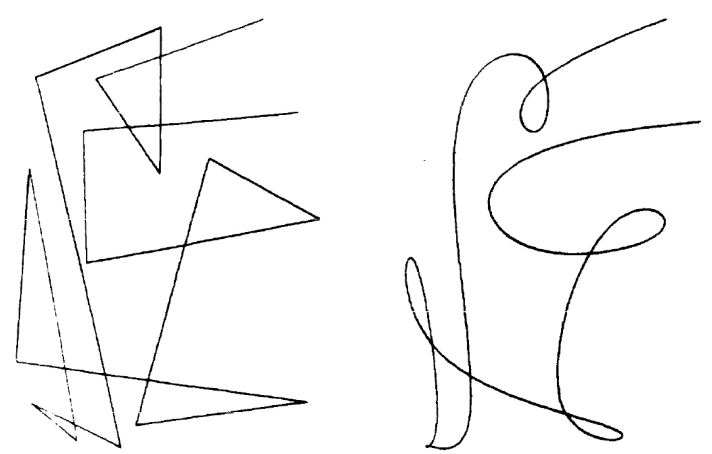

Fig.3a and 3b. showing the B-spline techniques for curve recognition and its "effects of varying the order of the spline". See figure 10 and 11 in "Recent advances in sketch recognition", (Negroponte, 1973)

HUNCH operated differently to its predecessor SKETCHPAD, designed in1963 by Ivan Sutherland. SKETCHPAD allowed "geometric shapes to be represented and conditions to be placed on these shapes in terms of constraints, to which they then conformed."(Sutherland, 1964) in (Simon, 1996) in (Stiny, 2006). SKETCHPAD was designed interpolate in order to 'draw' straight lines, it was not aimed at 'understanding' the user's pattern of drafting behavior; it was designed to understand the shape. George Stiny refers to Herbert Simon's understanding that "shapes 
First published in Werner, L. C. HUNCH 1972: A Second Experiment in Sketch Recognition or: 'I Know the Concept of Your Concept of Interpolation. In: Marcos, Carlos L. (ed.): Graphic Imprints: The influence of Representation and Ideation Tools in Architecture. Proceedings XVII EGA International Publishing, 2018, p. 3-11. 978-3-319-93748-9 ISBN (print).

are represented (structured) and well behaved computers." (Stiny, 2006) HUNCH was sensitive to little bumps and irregularities. In understanding the users sketching pattern, it would place this 'pattern' onto the raw sketch and create rationalize it in order to become a shape-like typology.

\subsection{The Hardware of HUNCH}

The system HUNCH was a subset of THE Architecture Machine. It consisted of an ARDS (advanced remote display station), a Sylvania Tablet on which the architect would place the paper and sketch (Figure 4). The tablet then would transfer pressure and speed in form of digits in space $(\mathrm{X}, \mathrm{Y})$ to the mini computer Inter-data Model Five, removable disk storage, the time-sharing system MULTICS, IMLAC a display unit that present three-dimensional graphics as the representation of SEEK (Negroponte, 1972). HUNCH had an interface from data and digitized analog information to SEEK, which would then interact with the stacked cubes in the cybernetic glass box. At US\$40,000, the purchase price for all components with its $16 \mathrm{k}$ memory (Inter-data mini computer) and 512k removable disk storage was low.

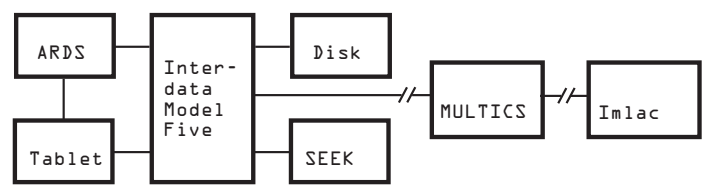

Fig. 4. shows the subset of the Architecture Machine used for HUNCH. Redrawn by author. Original can be found in "Recent advances in sketch recognition", (Negroponte, 1973)

\subsection{The Man-Machine System HUNCH}

The research project was presented at the annual conference on Environmental Design Research and AIA-Architect-Researchers held between January $24^{\text {th }}$ and $27^{\text {th }} 1972$ in Los Angeles; in Section 3 'Design - The Generation and Exploration of Solution' in the track 22 'Man-Machine Systems'. The conference and the subsequently published proceedings edited by William J. Mitchell featured more than 150 presentations and papers 1129 pages; discussing, presenting and engaging "with the current range of concerns in the emergent field of environmental design research" emphasizing "the relating of research to practice.”. (Mitchell, 1972) Topics included AI and ranged from design research and education via social and behavioral sciences. The presentation of HUNCH implied the question "what is design to emergent fields such as computer-aided design as a new approach to understand and handle challenges in complex systems such as environment and urban system or a novel man-machine relationship?'. In this respect conference Track 30 'Symposium on Conceptual Issues in Environmental Cognition Research' in Section 5 'Design Research and Education' seems particularly interesting;

a) when setting HUNCH's role in relationship with 'UDesign', and the ArchMac's agenda of training design software as well as the designer

b) in relation to an interdisciplinary approach in light of the attempt to apply a graphical Conversation Theory (Pask) to HUNCH.

Gary T. Moore, at that time, a visiting scholar at the Department of Psychology with a focus on behavioral sciences at Clark University, Massachusetts, chaired this track and defined that the "Environmental Cognition refers to the individuals or groups knowledge of the environment." (Moore, 1972) The conference benefited from the fact that the computer was still a new tool and instrument to explore. AI, computer sciences and the application of the digital calculation machine were supported by governmental and industrial financing bodies alike. The cold war situation helped to release funds and to strengthen political support for the first digital generation being curious and hungry to see what the machines can do and will do. HUNCH was one of the machines developed to see what it 'will' do rather than what it 'can' do.

\subsection{The vision of sketching having syntax}

Can a sketch be seen as the result of a coded syntax, owning grammar, rules, and pattern? Communication and translation of information are in many cases prone to error or faulty decoding, or complete misinterpretation unless the concept and the model of the concept are identical. In the case of $\mathrm{HUNCH}$, this is "when lines are formed at lower velocities, the interaction between the designer's concept and the 
First published in Werner, L. C. HUNCH 1972: A Second Experiment in Sketch Recognition or: 'I Know the Concept of Your Concept of Interpolation. In: Marcos, Carlos L. (ed.): Graphic Imprints: The influence of Representation and Ideation Tools in Architecture. Proceedings XVII EGA International Publishing, 2018, p. 3-11. 978-3-319-93748-9 ISBN (print).

machine's model of the concept appears to be most revealing. Crookedness appears, and there are uncertain gestures and highly reworked lines: this all may represent (perhaps semantic) dispositions toward a design such as being 'concerned about', sure of', 'puzzled by' and so forth." (Negroponte, 1972) HUNCH presented a crucial first step towards a digital acquaintance to the human designer, engaging and interacting with it, becoming a design partner who recognized syntactical patterns in a sketching process. It was developed to see what it will do when surrendering to the act of knowledge. $\mathrm{HUNCH}$, the fruit seeded by James Taggart had the goal of understanding a sketch created by a human, with all its bumpiness, crossing lines and open edges; designed with the human in mind - a humancentered design robot, that would look over the architects shoulder trying to understand, what the sketch meant geometrically and formally, but also semantically. Developed as a sketch recognition system, HUNCH was the system to discuss the questions if "drawing is a two-dimensional language, (and) does sketching have a syntax and semantics? Is any of HUNCH more than the syntactical processing of a hand drawing?" (Negroponte, 1975). The vision for HUNCH reached beyond merely being a sketch recognition software. HUNCH was anticipated to have a link, a comprehension of what we humans call the real world; it would understand human expressions and therefore be able to grow out of his school years as a graphics machine into his adult years, assisting humans with a multitude of inquiries.

\subsection{HUNCH as Design Tool for the layman}

HUNCH offered a number of research questions. One set of questions focused on the further development of computer/artificial intelligence, another on how to bring the tools of the architect closer to the layman, whose only option in the 1970 s was to buy a pre-fabricated house, designed without, as future inhabitant, having been able to participate or steer the design process. In a newspaper interview, published in the Chicago Tribute in 1974, Negroponte points out the common situation that "the only classes of people who get to design their homes are the rich in an industrialized society and the native in an underdeveloped society." (Shah,
1974) HUNCH was "a computer that would allow even the common old to draft a dream house." Everybody, not just the architect could have access to an instrument where he or she could design (at least) the layout of a dream home. On one hand, the system was equipped to help, assist and understand the ordinary person's individual visions, and on the other, it was a political act - to make the world a better place.

\section{CONCLUSION}

Despite that HUNCH like URBAN5 or U-Design proved to be a wonderful piece of kit to dive into the subject of conversing and designing in conjunction with a machine, the systems had their shortfalls. One was that the grade of complexity had its limits. Technically, HUNCH could only 'recognize' one user pattern during a session, and not several at the same time. A technical and solvable issue. During the time of $\mathrm{HUNCH}$, the computer had started to become a prosthetic, almost an extension of the architect. There is no question about the pencil being an extension of the architect already, the digital computer, however, has offered a further step using fast digital calculation and operation capacities; hence a shift in how we understand architecture comes about how the architectural design process is designed and could be designed in future; from object to process, from information exchange to form. Negroponte's vision encouraged architectural production to depart from a linear patriarch process to an interdisciplinary computational phylogenesis and the very shift we have been witnessing in the past 20 years; the transformation from a focus on objects to a focus on relationships. L'object d'architecture has morphed and mutated to l'object d'information. Architecture has always been an object of information, such as material properties or geothermal context, craftsmanship and skills or the daily operation of a social system that is living in the building. The kind of information, however, changes and develops. Nowadays we have grown categories of information that had no breeding ground before the digital computer, the invention of the Internet of smart materials. Information as material, an immaterial entity that is able to connect to open ends and insert into open systems, resulting in change. In the case of digitally produced architecture today, the immaterial information is about the building and embedded in the code of the geometry or performance optimization 
First published in Werner, L. C. HUNCH 1972: A Second Experiment in Sketch Recognition or: 'I Know the Concept of Your Concept of Interpolation. In: Marcos, Carlos L. (ed.): Graphic Imprints: The influence of Representation and Ideation Tools in Architecture. Proceedings XVII EGA International Publishing, 2018, p. 3-11. 978-3-319-93748-9 ISBN (print).

software; in the case of $\mathrm{HUNCH}$, the immaterial information was about the designer, the user, and his or her way of designing and sketching. The system extended the designer's closed conversation loop from two participants (designer and sketch) to three: designer, sketch and an environment, the sketch recognition system, which would 'remember' the designer's behavioral patterns.

\section{REFERENCES}

Brown, G. S. 1969. The Laws of Form, London, George Allen \& Unwin Lrd.

Foerster, H. v. 1979. Cybernetics of Cybernetics. In: Krippendorf, K. (ed.) Communication and Control. New York: Gordon and Breach.

Lynn, G. 2013. Archaeology of the Digital, Sternberg Press.

Mead, M. Cybernetics of Cybernetics. In: Foerster H. v., Peterson L. J., Russel J. K. , ed. Purposive Systems: proceedings of the first annual symposium of the American Society for Cybernetics, 1968. Spartan Books, 1-11.

Mitchell, W. J. 1972. Environmental Design: Research and Practice, Volumes One and Two. In: Mitchell, W. J., ed. Environmental Design Research Association and AIA-Architect-Researchers' Annual Conference, 1972 University of California, Los Angeles. Environmental Design Research Association.

Moore, G. T. Conceptual Issues in the Study of environmental Cognition. An Introduction. In: Mitchell, W. J., ed. Environmental Design Research Association and AIA-Architect-Researchers' Annual Conference, 1972 University of California, Los Angeles. Environmental Design Research Association.

Negroponte, N. Recent advances in sketch recognition. Proceedings of the June 4-8, 1973, national computer conference and exposition, 1973. ACM, 663-675.

Negroponte, N. 1975. Soft Architecture Machine, Cambridge, MA, The MIT Press.

Negroponte, N., Groisser, L. B.. Taggart, J..
HUNCH: An Experiment in Sketch Recognition. In: Mitchell, W. J., ed. Environmental Design research Association and AIA-Architect-Researchers' Annual Conference, 1972 University of California, Los Angeles. Environmental Design Research Association.

Pask, G. 1976. Conversaton Theory - Applications in Education and Epistomolgy, Amsterdam, Elsevier Scientific Publishing Company.

Shah, D. K. 1974. Computer may build a house next. Chicago Tribune.

Simon, H. A. 1996. The sciences of the artificial, MIT Press. pp. 134-35.

Stiny, G. 2006. Shape: talking about seeing and doing, MIT Press.

Sutherland, I. E. 1964. Sketchpad a man-machine graphical communication system. Transactions of the Society for Computer Simulation, 2, R-3-R-20.

Vardouli, T. 2011. Nicholas Negroponte: an interview [Online]. open | architectures: computational making group, MIT architecture. [Accessed 20180110].

Werner, L. C. forthcoming. The Origins of Design Cybernetics. In: Fischer, T., Herr, C. M. (ed.) Design Cybernetics: Navigating the New. Springer. 